\title{
RTK GPS alıcısının yön açısı ölçüm doğruluğunun dijital pusula yardımı ile belirlenmesi
}

\section{Determining the heading angle measurement accuracy of RTK GPS receiver by the help of digital compass}

\author{
İlker ÜNAL \\ Akdeniz Üniversitesi, Teknik Bilimler Meslek Yüksekokulu, 07070, Antalya \\ Sorumlu yazar (Corresponding author): İ. Ünal, e-posta (e-mail): ilkerunal@akdeniz.edu.tr
}

MAKALE BİLGISİ

Alınış tarihi 05 Ekim 2020

Düzeltilme tarihi 27 Ekim 2020

Kabul tarihi 27 Ekim 2020

\section{Anahtar Kelimeler:}

Otonom tarımsal robotlar

Navigasyon

Yön açısı

Topcon RTK GPS alıcisı

HMR 3000 dijital pusula

\begin{abstract}
ÖZ
Otonom tarımsal mobil robotlar, tarımsal işlemler için traktörlere bir alternatiftir. Ancak, bu robotların dikim, ilaçlama, gübreleme ve hasat gibi farklı tarımsal görevlerde sağlam ve güvenilir bir navigasyon sistemine ihtiyacı bulunmaktadır. Bu bağlamda, hedef noktalara doğru ve hızlı dönüş manevraları gerçekleştirmek için dijital bir pusula veya bir GPS alıcısından robot yön açısı ölçümleri, hassas tarım robotu navigasyon algoritmaları için en önemli prosedürdür. Bu çalışmanın amacı, Honeywell HMR3000 dijital pusula yardımıyla Topcon RTK GPS alıcısının yön açısı ölçüm doğruluğunu ve aynı zamanda her iki cihaz arasındaki düzeltme faktörünü belirlemektir. Bu çalışmada, robotun kuzeye göre yönünü ölçmek için Honeywell HMR3000 dijital pusula kullanılmıştır. Bu veriler, Topcon RTK GPS alıcısının rota açısı ölçümlerinin nicel korelasyonunu oluşturmak için kalibrasyon veri seti olarak kullanılmıștır. Çalıșma sonucunda, Topcon RTK GPS alıcısı ile HMR3000 arasında yüksek oranda doğrusallık olmasına rağmen $\left(\mathrm{R}^{2}=0.9998\right)$, açı değerlerinin -5.87 ile +5.97 derece arasında saptığı gözlemlenmiştir. Bu sonuç, GPS alıcısından elde edilen yön açısı değerlerinin hassas navigasyon uygulamaları için kullanılabilir olmadığını göstermektedir.
\end{abstract}

\section{ARTICLE INFO}

Received 05 October 2020

Received in revised form 27 October 2020

Accepted 27 October 2020

\section{Keywords:}

Autonomous agricultural robots

Navigation

Heading angle

Topcon RTK GPS receiver

HMR 3000 digital compass

\begin{abstract}
Autonomous agricultural mobile robots are an alternative to the tractors for cultivation tasks But, these robots need a robust and reliable navigation system in different agricultural tasks such as planting, spraying, fertilizing, and harvesting. In this context, the robot heading angle measurements from a digital compass or a GPS receiver to perform accurate and rapid turning manoeuvres toward the target points are the most important procedure for precise agricultural robot navigation algorithms. The objective of this study was to determine the heading angle measurement accuracy of Topcon RTK GPS receiver by the help of Honeywell HMR 3000 digital compass. It is also to determine the correction factor between both devices. In this study, Honeywell HMR3000 digital compass was used to measure the direction of the robot relative to North. These data were used as calibration dataset to establish the quantitative correlation the heading angle measurements of the Topcon RTK GPS receiver. As a result of the study, although there is a high level of linearity between the Topcon RTK GPS receiver and the HMR3000 $\left(\mathrm{R}^{2}=0.9998\right)$, it was observed that the angle values deviated between -5.87 and +5.97 degrees. This result shows that the direction angle values obtained from the GPS receiver are not usable for precise navigation applications.
\end{abstract}

\section{Giriş}

Tarımsal amaçlı otonom bir robot, tarımsal üretim ortamında sürücüsüz olarak hareket edebilen tekerlekli bir araç olarak tanımlanmaktadır. Burada kullanılan otonom ifadesi, robot üzerine yerleştirilen dâhili veya harici sensörler yardımıla robotun ortam hakkında zamansal ve mekânsal veriler toplayarak hareket etme ve yönlenme yeteneği olarak ifade edilmektedir. Otonom bir robot, tarımsal üretim ortamında hareket edebilme ve yönlenme işlevlerini motorlu hareketlendiriciler yardımıyla, üzerine yerleştirilen bilgisayara önceden yüklenmiş olan referans veriler ve sensörlerden toplanan veriler üzerinde farklı algoritmalar kullanarak gerçekleştirmektedir (Li ve ark. 2009). Özellikle açık tarla ortamında kullanılan robotların belirlenen hedef noktalara yönlendirilmesi ve doğrusal hareketlerinin sağlanabilmesi için 
GPS (Global Positioning System) alıcıs1 ve dijital pusulalar en önemli sensörler olarak kullanılmaktadır.

Robot navigasyon uygulamalarında başarılı olunabilmesi için doğru yön ve konum verilerinin hesaplanması gerekmektedir. GPS, doğru navigasyon verileri sağlama konusundaki üstün yetenekleri nedeniyle birçok bilimsel ve mühendislik uygulamalarında kullanılmaktadır. Bir noktanın dünya üzerindeki gerçek konumunun belirlenmesi, sadece kullanılan GPS alıcısına değil, konum verilerinin hassasiyetini gerçek zamanlı $\mathrm{cm}$ mertebesinde belirleyen klasik RTK (Gerçek Zamanlı Kinematik) veya CORS (Continuously Operating Reference Station) tekniklerinin kullanılmasına bağlıdır (Keicher ve Seufert 2000; Mizushima ve ark. 2003). Bir GPS alıcısı kullanılan düzeltme teknikleri yardımıyla hassas konum belirleyebilmesine rağmen, navigasyon işleminin bir diğer parametresi olan yön açısını (heading) uydu sinyallerini alamadığı ya da durağan olduğu durumlarda doğru belirleyememektedir. $\mathrm{Bu}$ bağlamda, bir navigasyon uygulamasının gerçekleştirilebilmesi için GPS alıcısı ile birlikte robotun yön açısını güvenli şekilde belirleyebilen dijital bir pusulaya ihtiyaç bulunmaktadır. GPS alıcısı ve dijital pusulanın entegrasyonundan elde edilen konum ve yön bilgileri, mobil bir robotun noktadan noktaya doğru bir şekilde yönlendirilmesinde kullanılacak olan navigasyon algoritmasinın temel referans verilerini oluşturmaktadırlar (Ryu ve ark. 2016). Yön açıs1, yatay bir düzlemde hareket eden bir aracın boylamsal eksen doğrultusu ile kuzey yönü arasında kalan açı olarak tanımlanmaktadır (Şekil 1). Robot navigasyon uygulamalarında yön açısı çoğu zaman robotun hareket yönü olarak kullanılmaktadır. Yön açısı verisi, robotun hem bilinen önceki konum bilgileri ile birlikte kullanıldığında mevcut konumunu belirlemek, hem de bilinen hedef bir noktaya yönlenmesinde gerekli olan dönüş açısının hesaplamada kilit bir rol oynamaktadir.

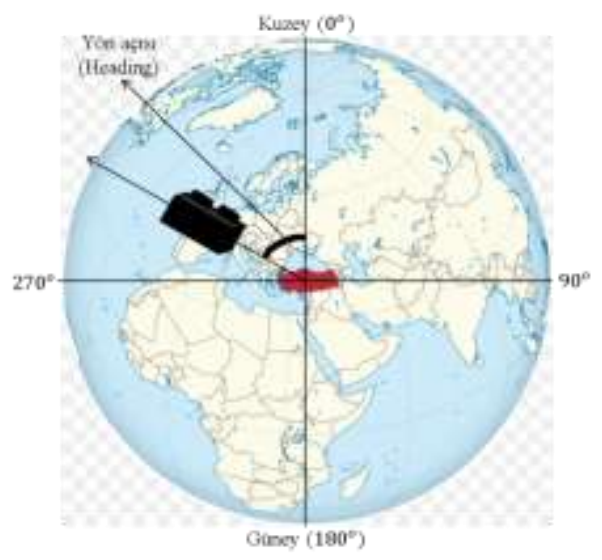

Şekil 1. Yön açısı.

Figure 1. Heading angle.

Literatürde, tarımsal amaçlı otonom robotların sadece GPS alıcıs1 kullanılarak yönlendirilmesi üzerine yapılmış bazı çalıșmalar bildirilmiștir (Stoll ve Kutzbach 2000; Thuilot ve ark. 2001). Ancak, tek bir GPS alıcis1 kullanılarak gerçekleştirilen otonom navigasyon uygulamaları bazı sinırlamalara sahiptir. Bu sinırlamaların giderilmesi ve daha doğru navigasyon bilgileri sağlamak için İnersiyal Ölçme Ünitesi (IMU - Inertial Measurement Unit) ile birleştirilmesi gerekmektedir (Hellström 2002). Son 10 yıllık süreçte GPS ve dijital pusulanın navigasyon sistemleri içerisinde entegreli şekilde kullanımı ile birbirlerini tamamladıkları görülebilmektedir. Zhang ve ark. (2015), bir biçerdöverin IMU entegreli bir RTK GPS kullanılarak konum ve duruş verileri üzerinden otonom şekilde harita tabanlı bir navigasyon algortiması ile kontrolünü gerçekleştirmişlerdir. Oksanen ve Backman (2013), otonom olarak çalıştırılabilecek şekilde tasarlanmış bir traktör için navigasyon algoritması geliştirmişlerdir. Çalışmada, konum belirlemek için RTK GPS alıcısı, yön açısı belirlemek için ise fiber optik jiroskop kullanmışlardır. Yön açısı belirleme algortimasında hem jiroskop hem de GPS alıcısından gelen yön açısı verileri beraber kullanılmıştır. Han ve ark. (2017), traktörlerin otonom şekilde navigasyonunun sağlanması için traktör üzerine üçgen oluşturacak şekilde yerleştirilen üç adet düşük maliyetli GPS alıcısı ve IMU içeren entegre bir sistem geliştirmişlerdir. Diğer yandan, Gomez-Gil ve ark. (2011), otonom navigasyon uygulamalarında yönlendirme hatalalarının azaltılması için daha hassas GPS alıcıları, diferansiyel düzeltmeler ve elektronik pusula kullanılması gerektiğini ancak bu sistemlerin pahalı çözümler olduğunu belirtmişlerdir. Araştırmacılar çalışmalarında, IMU sistemi kullanmadan, traktörden daha ileri bir konuma yerleştirilen tek bir GPS alıcısından alınan konum verileri ve traktör kinematik yasalarını da hesaba katarak traktör yön değişikliklerini belirlemek için basit ve düşük maliyetli bir yöntem geliştirmişlerdir.

Günümüzde birçok araştırmacı daha hassas hareket edebilen tarımsal amaçlı robot tasarımlarında sensör füzyonu teknolojisine odaklanmışlardır. Sensör füzyonunda, birden fazla sensörden gelen analog ve dijital verilerin amaca uygun şekilde değerlendirilip robot navigasyon uygulamalarında daha hassas ve esnek sonuçlar elde edilmesi amaçlanmaktadır. Ancak, herbir teknoloji yapılan çalışmaların maliyetini arttırmaktadır. Bu çalışmada, düzeltme sinyallerini CORS üzerinden alan tek bir RTK GPS alıcısından elde edilen yön açısı verilerinin navigasyon uygulamalarında kullanım imkanını arttıran bir yöntem açıklanmıştır. Bu amaçla, asıl kullanım amacı yön açısı bulmak olan dijital pusuladan elde edilen veriler ile GPS alıcısından elde edilen yön açısı verilerinin kalibrasyonunu sağlayacak olan düzeltme faktörünü belirlemeye çalışılmıştır.

\section{Materyal ve Yöntem}

\subsection{Materyal}

$\mathrm{Bu}$ çalışmada, coğrafi konum ve yön açılarının toplanması için önceki çalışmalarda oluşturulmuş olan tarımsal amaçlı otonom bir mobil robot kullanılmıştır. Mobil robotun otonom çalıştırılması sırasında geçilen noktalara ait coğrafi konum (enlem, boylam) ve yön açılarının (heading) toplanması için Topcon AGI-4 çift kanallı (L1, L2) RTK GPS alıcıs1 kullanılmıştır.

GPS alıcısının katalog verilerine göre RTK ölçüm hassasiyeti $2 \mathrm{~cm}$ altı olarak belirtilmiştir. GPS alıcısı için düzeltme sinyalleri CORS-TR ağından alınmıştır. GPS alıcısı ile eş zamanlı olarak mobil robotun yön açısını toplamak için Honeywell HMR3000 dijital pusula kullanılmıştır.

Dijital pusulanın katalog verilerine göre yön açısı ölçümünde 0.1 derece çözünürlükte 0.5 dereceden daha az ölçüm hassasiyetine sahip olduğu belirtilmiştir. GPS alıcısı ve dijital pusuladan gelen verileri toplamak için ECS-4500 endüstriyel bilgisayar kullanılmıştır. GPS alıcısı ve dijital pusula endüstriyel bilgisayara RS232 seri iletişim portu üzerinden bağlanmıştır. 


\subsection{Metot}

Çalışmada, GPS alıcısı ve dijital pusula mobil robot ekseni üzerinde aynı doğrultuda ve $50 \mathrm{~cm}$ aralıklı olacak şekilde yerleştirilmiştir. Belirtilen bu mesafe dijital pusulanın, GPS alıcısının manyetik alan etkisinden uzaklaşmak için belirlenmiştir (Şekil 2). GPS alıcısı ve dijital pusulanın bilgisayar ile seri haberleşme hızı 19200 baud olarak ayarlanmıştır. GPS alıcısının verileri yenileme hızı $10 \mathrm{~Hz}$, dijital pusulanın ise $20 \mathrm{~Hz}$ olacak şekilde ayarlanmıştır. Bu frekanslar, kullanılan cihazlara ait maksimum değerlerdir. Veri toplama yazılımı, Microsoft Visual Basic.NET programlama dilinde geliştirilmiştir. Tüm veriler, Microsoft SQL Server 2005 veri tabanına saniyede bir olacak şekilde kaydedilmiştir. Yön açısı verilerini analiz etmek için ise Microsoft Excel 2010 kullanılmıştır.

Veri toplama çalışmaları, Akdeniz Üniversitesi kampüsü içerisinde, yönü güney-kuzey doğrultuda $36^{\circ} 53^{\prime} \mathrm{N}$ ve $30^{\circ} 38^{\prime} \mathrm{E}$ koordinatlarında bulunan tali bir yol üzerinde gerçekleştirilmiştir. GPS alıcıları hesapladıkları değişkenleri (konum, hız, yön açısı gibi) RS232 portu üzerinden bağlı oldukları bilgisayara aktarmak için Amerika Ulusal Deniz Elektroniği Birliği (NMEA) tarafından standart hale getirilmiş olan NMEA - 0183 veri formatını kullanırlar. Çalışmada, GPS alıcısının 100 ms aralıklarla geliştirilen veri toplama yazılımına NMEA - 0183 veri format içerisinde bulunan "\$GPRMC" veri kümesini göndermesi sağlanmıștır. "\$GPRMC" veri kümesi, araları birbirinden virgüllerle ayrılmış 11 farklı veri içermektedir. $\mathrm{Bu}$ veriler içerisinde bulunan enlem, boylam verileri derece-dakika formatında olması nedeniyle UTM formatına çevrilerek metrik olarak ölçülendirilmiş ve veri tabanına kaydedilmiştir. Veri kümesi içerisindeki yön açısı verisi ise olduğu gibi derece formatında veritabanına kaydedilmiştir. HMR3000 dijital pusula da aynı şekilde yön verisini RS232 portu üzerinden bağlı olduğu bilgisayara NMEA - 0183 veri formatını kullanarak göndermektedir. GPS alıcısı ve dijital pusulanın göndermiş olduğu NMEA - 0183 verileri Çizelge 1'de gösterilmiştir.

Çalışma esnasında, GPS alıcısından gelen enlem, boylam ve yön açısı ile dijital pusuladan gelen yön açısı verileri Microsoft Visual Studio 2015 içerisinde bulunan Visual Basic.NET platformunda geliştirilen veri yakalama programı ile Microsoft SQL Server 2005 veri tabanı içerisine aktarılmıştır. Geliştirilen yazılıma ait ekran görüntüsü Şekil 3'de gösterilmiştir.

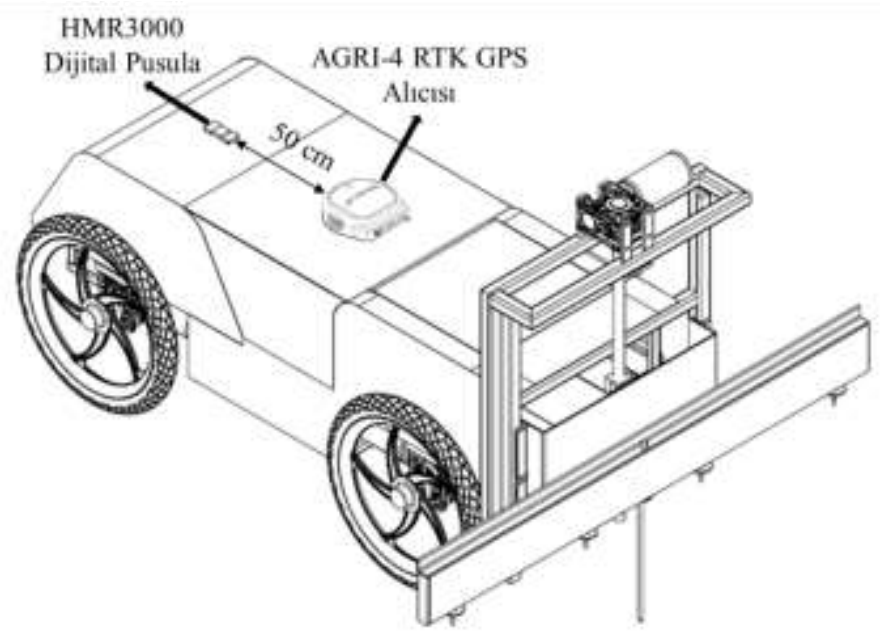

Şekil 2. Mobil robot ve sensörlerin yerleşimi.

Figure 2. The settlement of the mobile robot and sensors.

Çizelge 1. GPS alıcısı ve dijital pusulaya ait veri formatları.

Table 1. Data format of the GPS receiver and digital compass.

\begin{tabular}{|c|c|c|}
\hline Sensör & Veri Format1 & Tanımlamalar \\
\hline $\begin{array}{l}\text { AGI-4 RTK GPS } \\
\text { Alıcis1 }\end{array}$ & $\begin{array}{l}\text { \$GPRMC, HHMMSS.SS, Q, LLLL.LL, A, YYYYY.YY, A, } \\
\text { X.X, X.X, XXXX, X.X, A*HH }\end{array}$ & $\begin{array}{l}\text { HHMMSS.SS: Zaman (UTC) } \\
\text { Q: Durum } \\
\text { LLLL.LL: Enlem } \\
\text { A: N veya S } \\
\text { YYYYY.YY: Boylam } \\
\text { A: E veya W } \\
\text { X.X: Ilerleme hızı, knots } \\
\text { X.X: Yön açıs, derece } \\
\text { XXXX: Tarih, ddmmyy } \\
\text { X.X: Manyetik Sapma, derece } \\
\text { A: E veya W } \\
\text { HH: Kontrol }\end{array}$ \\
\hline $\begin{array}{l}\text { HMR3000 Dijital } \\
\text { Pusula }\end{array}$ & \$HCHDT,X.X,T*HH<CR $><L F>$ & $\begin{array}{l}\text { X.X: Yön (Heading) açısı, derece } \\
\text { T*HH: True, Kontrol } \\
\text { CR: Satır Sonu } \\
\text { LF: Enter }\end{array}$ \\
\hline
\end{tabular}




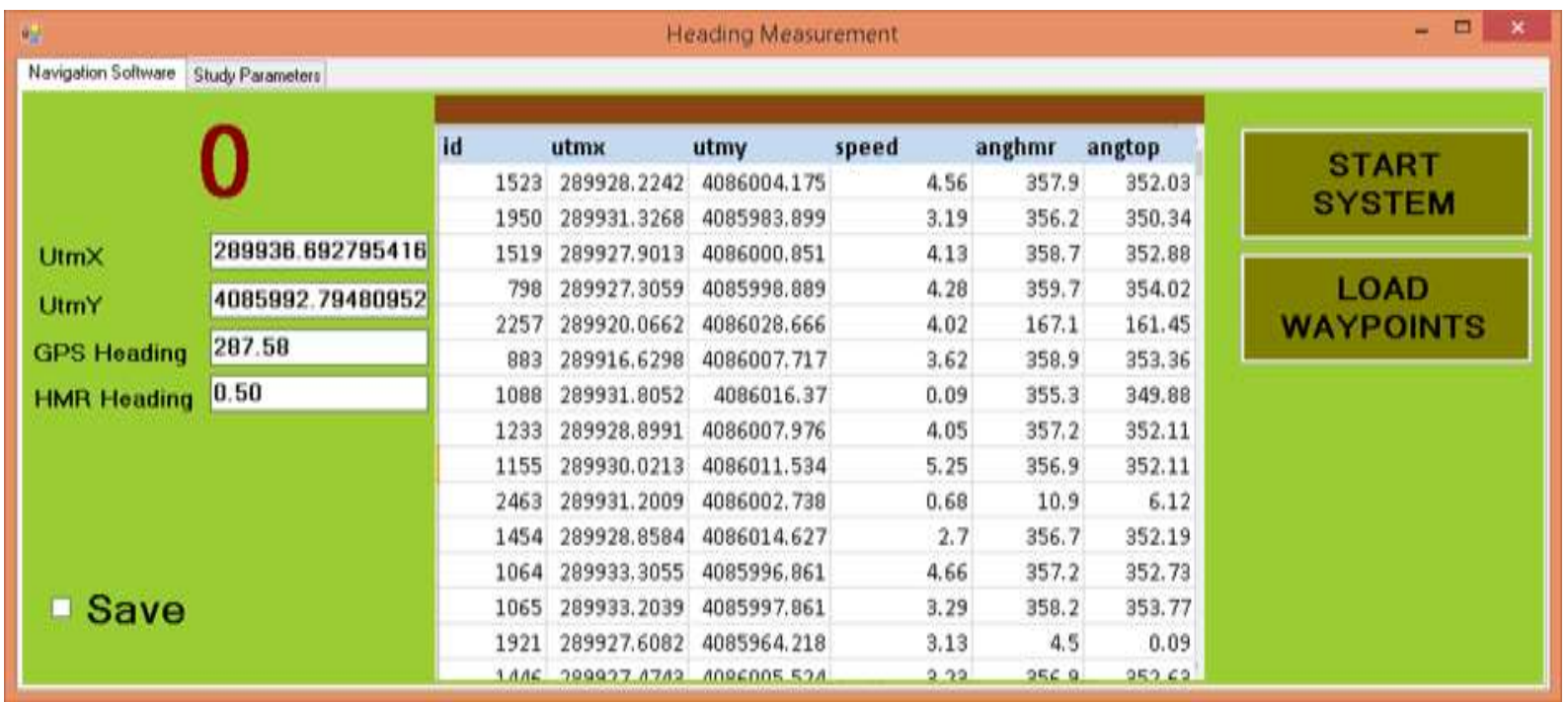

Şekil 3. Veri toplama yazılımı ekran görüntüsü.

Figure 3. The screens hot of the data acquisition software.

Hem GPS alıcısı, hem de dijital pusuladan elde edilen yön açısı verileri üzerinde istatistiksel analiz yapmak için Microsoft Excel 2010 programı kullanılmıştır. Literatürde, iki farklı sensörden alınan benzer veriler üzerindeki ilişkisel benzerlik ya da farklılıkları belirlemek için birçok istatistiksel yöntem önerilmiştir. Bu çalışmada, iki veri arasındaki ilişkiyi açıklamak ve hassasiyet ve doğruluğu kesin olarak bilinen dijital pusuladan elde edilen yön verileri ile GPS verilerinin düzeltme katsayısını belirlemek için lineer regresyon, Ortalama Kare Hata (MSE), Ortalama Karekök Hata (RMSE) ve Ortalama Mutlak Hata (MAE) yöntemleri kullanılmıştır.

$$
\begin{aligned}
& M S E=\sum_{t=1}^{N}\left(\frac{\theta_{g p s}-\theta_{h m r}}{N}\right)^{2} \\
& R M S E=\sqrt{\frac{1}{N}\left[\sum_{t=1}^{N}\left(\frac{\theta_{g p s}-\theta_{h m r}}{\theta_{g p s}}\right)^{2}\right]} \\
& M A E=\frac{1}{N} \sum_{t=1}^{N}\left|\theta_{g p s}-\theta_{h m r}\right|
\end{aligned}
$$

Burada, $\theta_{g p s}$ : GPS alıcısından alınan yön açısı, $\theta_{h m r}$ : Dijital pusuladan alınan yön açısı, N: toplam veri sayısı olarak tanımlanmaktadır.

\section{Bulgular ve Tartışma}

Çalışma süresince, mobil robot otonom şekilde farklı noktalara yönlendirilmiştir. GPS alıcısı ve dijital pusuladan eş zamanlı olarak toplam 239 adet konum ve yön açısı verileri kayıt altına alınmıştır. Dijital pusuladan alınan 239 adet yön verisi doğrulama veri seti olarak kullanılmıştır. GPS alıcısından elde edilen yön verileri ise, dijital pusuladan ele edilen verilere göre Microsoft Excel 2010 programı kullanılarak istatistiksel olarak analiz edilmiştir. Standart olarak gerçekleştirilen istatistiksel analizlerde veriler arasındaki matematiksel farkın negatif olmasına bakılmaksızın sonuçlar hesaplanmaktadır. Çizelge 2'de toplanan yön açısı verilerine göre, MAE, MSE, RMSE ve $\mathrm{R}^{2}$ değerleri gösterilmiştir. Ayrıca, Şekil 4'de doğrusal regresyon grafiği gösterilmiştir.

Çizelge 2. Standart olarak yapılan istatistiksel analiz sonuçları.

Table 2. Standard statistical analysis results.

\begin{tabular}{cccc}
\hline MAE & MSE & RMSE & R $^{2}$ \\
\hline 2.304 & 7.977 & 2.824 & 0.9998 \\
\hline
\end{tabular}

Yapılan alan çalışmada elde edilen 239 verinin 116 tanesinde veriler arasındaki matematiksel farkın negatif, 123 tanesinin ise pozitif olduğu gözlemlenmiştir. Analiz sonucunda $\mathrm{R}^{2}$ değerinin 0.9998 çıktığ 1 görünse bile GPS alıcısından elde edilen açı verilerinin dijital pusulaya göre -5.87 ile +5.97 derece arasında saptı̆̆ gözlemlenmiştir. Bu sonuç, tek başına bir GPS alıcısının hassas mobil robot navigasyon uygulamalarında yön açısı ölçümü için uygun olmadığını göstermektedir. Ancak, her iki sensörün beraber kullanıldığı uygulamalarda dijital pusula verileri referans alınarak GPS alıcısından elde edilen yön açısı verilerinin mümkün mertebe düzeltilebileceği ve navigasyon uygulamalarında sistem için geri besleme elemanı olarak kullanılabileceği düşünülmektedir. Her ne kadar dijital pusulaların yüksek ölçüm hassasiyetine sahip oldukları bilinmekle birlikte, en küçük bir manyetik alan değişiminden olumsuz olarak etkilenmektedirler. $\mathrm{Bu}$ bağlamda, dijital pusulaların ortamdaki manyetik alan değişiminin ortadan kalkmasına kadar geçen süre içerisinde yön açısı verilerinin GPS alıcısından gelen açı verileri ile optimize edilebileceği öngörülmektedir. Bu kapsamda çalışmada, GPS alıcısı yön açısı verileri için bir açı düzeltme algoritması önerilmiştir. Algoritmaya ait akış şeması Şekil 5'de verilmiştir.

GPS alıcısından elde edilen veriler için düzeltme katsayısının belirlenmesinde her iki sensör arasındaki negatif farkların toplamının ortalaması GPS alıcısından elde edilen yön açısı verisine yinelemeli şekilde eklenerek, pozitif farkların toplamının ortalaması ise çıkarılarak veriler yeniden istatistiksel olarak analiz edilmiştir. Analiz sonucunda $\mathrm{R}^{2}$ değerinin 0.9999'a çıktığı ve GPS alıcısından elde edilen açı verilerinin dijital pusulaya göre -3.59 ile +3.65 derece arasında saptı̆̆ 1 
gözlemlenmiştir. Düzeltme katsayısı hesaplama algoritması sonrası elde edilen istatistiksel analiz sonuçları Çizelge 3 'de gösterilmiştir.

Çizelge 3. Düzeltme katsayısı eklendikten sonra yapılan istatistiksel analiz sonuçları.

Table 3. Statistical analysis results after adding the correction coefficient.

\begin{tabular}{cccc}
\hline MAE & MSE & RMSE & $\mathrm{R}^{2}$ \\
\hline 1.367 & 2.661 & 1.631 & 0.9999 \\
\hline
\end{tabular}

Çalışmada kullanılan dijital pusula hassas ölçüm yapma noktasında, piyasada bulunan diğer pusulalara göre ortalamanın üzerinde bir sensördür. Sensörün güncel fiyatı, yaklaşık olarak
1000 \$ civarındadır. Ölçüm sensörlerinde doğruluğu etkileyen iki önemli faktör bulunmaktadır. Bunlardan birincisi ölçüm hassasiyeti diğeri ise anlık veri gönderme hızıdır. Özellikle sensör tabanlı sistemlerde ölçüm hassasiyeti ve anlık veri gönderme hızının yüksekliği, kullanılan sensörün edinme maliyeti ile doğru orantılıdır. Bu açıdan bakıldı̆̆ında, hassas navigasyon uygulamalarında sensörlerin edinme maliyetinden ziyade hassasiyet ve veri gönderme hızının yüksekliği, yapılacak olan çalışmanın başarısı açısından önem arz etmektedir. Bu bağlamda, hassas navigasyon uygulamalarında uygun fiyatlı dijital pusulaların piyasada hassas olarak kabul edilen referans bir pusula ile karşılaştırılarak kullanılabilirliğinin araştırılması gerekmektedir.

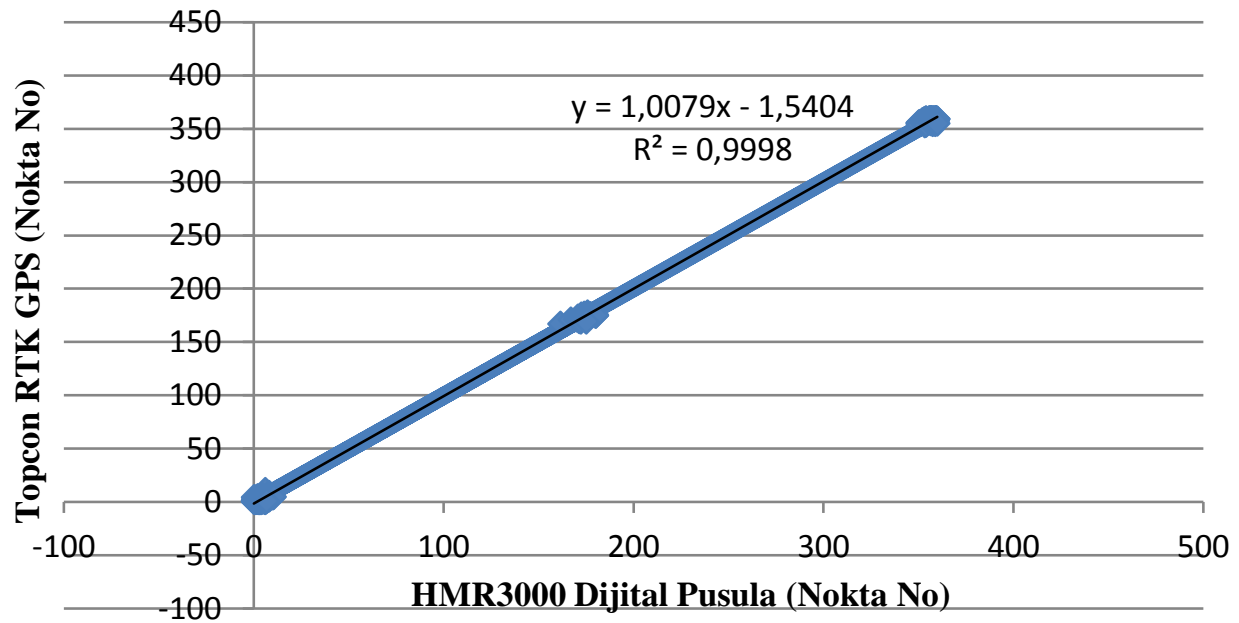

Şekil 4. Yön açısı verilerinin doğrusal regresyon grafiği

Figure 4. Linear regression plot of heading angle data

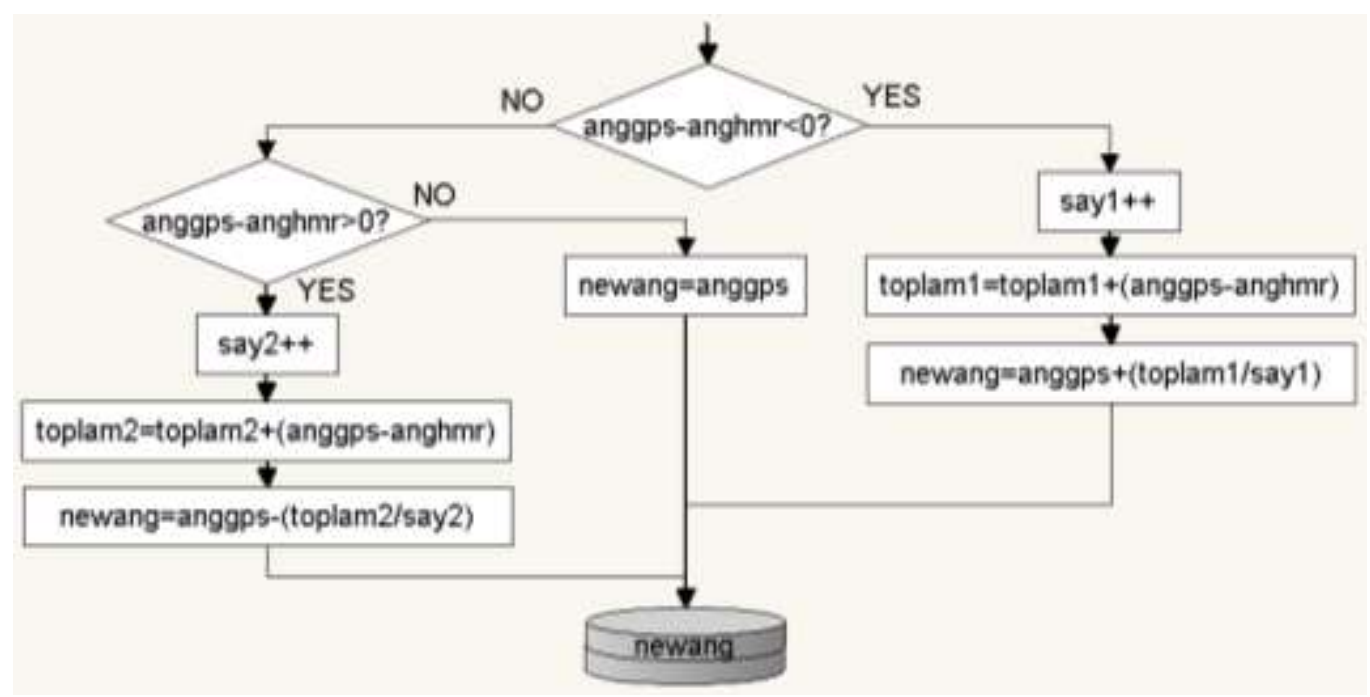

Şekil 5. GPS alıcısı açı verileri için önerilen düzeltme katsayısı hesaplama akış şeması

Şekil 5. The flowchart of suggested correction coefficient calculation for GPS receiver angle data 


\section{Sonuç}

Bu çalışmada, Topcon AGI-4 RTK GPS alıcısının mobil robotlar için yön açısı belirlenmesinde kullanılabilirliği tartışılmıştır. Yön açısı ölçüm hassasiyetinin belirlenebilmesi için ölçüm hassasiyeti yüksek olan HMR3000 dijital pusula verilerine göre GPS alıcısı verileri karşılaştırılmıştır. Yön açısı, mobil robotların bir noktadan başka bir noktaya otonom olarak yönlendirilirken sağa ya da sola dönüşlerindeki manevraları etkileyen en önemli navigasyon parametresidir. Her ne kadar RTK GPS alıcılarının hareketli konum hassasiyetleri santimetre mertebesinde olsa bile yön açısı ölçüm hassasiyetlerinin de belirlenmesi gerekmektedir. Bu kapsamda, yapılan çalışmada her iki sensör arasında yüksek oranda doğrusallık olmasına rağmen, GPS alıcısından elde edilen yön açısı verilerinin hassas navigasyon uygulamalarında tek başına bir açı sensörü olarak kullanılamayacağını göstermiştir. Ancak, dijital pusula ile birlikte oluşturulacak bir IMU sistemi için çalışmada önerilen düzeltme algoritması kullanılarak belli bir seviyeye kadar sistem geri besleme elemanı olarak kullanılabileceği düşünülmektedir.

\section{Kaynaklar}

Gomez-Gil J, Alonso-Garcia S, Gómez-Gil FJ, Stombaugh T (2011) A Simple Method to Improve Autonomous GPS Positioning for Tractors. Sensors 11: 5630-5644.

Han XZ, Kim HJ, Jeon CW, Moon HC, Kim JH (2017) Development of a low-cost GPS/INS integrated system for tractor automatic navigation. International Journal of Agricultural and Biological Engineering 10(2): 123-131.

Hellström T (2002) Autonomous navigation for forest machines, A project pre-study in the Department of Computer Science Umea University, Sweden.

Keicher R, Seufert H (2000) Automatic guidance for agricultural vehicles in Europe. Computers and Electronics in Agriculture 25(1): 169-194.

Li M, Imou K, Wakabayashi K, Yokoyama S (2009) Review of research on agricultural vehicle autonomous guidance. International Journal of Agricultural \& Biological Engineering 2(3): 1-16.

Mizushima A, Noguchi N, Ishii K, Terao H (2003) Development of navigation sensor unit for the agricultural vehicle. In Proceedings of the Advanced Intelligent Mechatronics, Kobe, Japonya.

Oksanen T, Backman J (2013) Guidance system for agricultural tractor with four wheel steering. In: The IFAC bio-robotics conference. Osaka, Japonya.

Ryu JH, Gankhuyag G, Chong KT (2016) Navigation System Heading and Position Accuracy Improvement through GPS and INS Data Fusion. Journal of Sensors 2016: 1-6.

Stoll A, Kutzbach HD (2000) Guidance of a forage harvester with GPS. Precision Agriculture 2: 281-291.

Thuilot B, Cariou C, Cordesses L, Martinet P (2001) Automatic guidance of a farm tractor along curved paths, using a unique CPDGPS. Proceedings of the 2001 IEEE/RSJ International Conference on Intelligent Robots and Systems, Maui, ABD.

Zhang Z, Noguchi N, Ishii K (2015) Development of a Robot Combine Harvester. Journal of the Japanese Society of Agricultural Machinery and Food Engineers 77(1): 45-50. 